
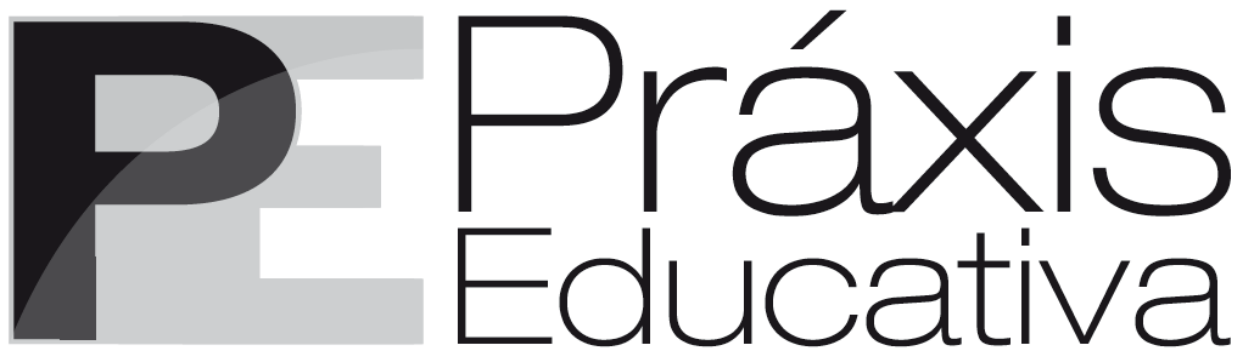

ISSN 1809-4031

elSSN 1809-4309

https://doi.org/10.5212/PraxEduc.v.16.15143.005

\title{
Los viajes de Paulo Freire a España (1984-1994) y la consolidación del ámbito académico de la pedagogía social a partir de los años noventa
}

\section{Viagens de Paulo Freire à Espanha (1984-1994) e a consolidação do campo acadêmico de pedagogia social dos anos noventa}

\section{Paulo Freire's journeys to Spain (1984-1994) and the consolidation of the academic field of social pedagogy from the $90 \mathrm{~s}$}

\author{
Jon Igelmo Zaldívar \\ https://orcid.org/0000-0002-8587-2822 \\ Patricia Quiroga Uceda* \\ iD https://orcid.org/0000-0001-7435-0220
}

\begin{abstract}
Resumen: Las ideas de Paulo Freire llegaron a España a partir de los años setenta. Los primeros espacios de recepción fueron los proyectos de educación popular que estaban siendo impulsados por sectores progresistas de la Iglesia y también por los Movimientos de Renovación Pedagógica. A finales de los años ochenta y principios de noventa las ideas de Freire fueron recibidas en el ámbito académico de la pedagogía. Los viajes que Freire realiza a España entre 1984 y 1994, cuatro viajes que son estudiados con detalle en este artículo, son una fuente histórica clave para este estudio de la presencia del pensamiento del pedagogo brasileño en las Facultades de Educación españolas. La hipótesis central que desarrollamos en este trabajo es que tomando como referencia la obra de Freire la pedagogía social consiguió cavar su propio espacio en el campo académico de la pedagogía en España.
\end{abstract}

Palabras clave: Paulo Freire. España. Pedagogía social. Educación Popular.

Resumo: As ideias de Paulo Freire chegaram à Espanha a partir da década de 1970. Os primeiros espaços de acolhimento foram os projetos de educação popular promovidos por setores progressistas da Igreja e também pelos Movimentos Pedagógicos de Renovação. As ideias de Freire foram recebidas no campo acadêmico da pedagogia no final dos anos 80 e início dos anos 90. As viagens de Freire à Espanha entre 1984 e 1994, quatro viagens que são estudadas em detalhes neste artigo, são uma fonte histórica fundamental

\footnotetext{
* Profesor Ayudante Doctor del Departamento de Estudios Educativos de la Facultad de Educación de la Universidad Complutense de Madrid (UCM) y co-director del grupo de investigación Cultura Cívica y Políticas Educativas. E-mail: <jigelmoz@ucm.es>.

** Profesora Ayudante Doctor del Departamento de Estudios Educativos de la Facultad de Educación de la Universidad Complutense de Madrid (UCM) y miembro del grupo de investigación Cultura Cívica y Políticas Educativas. E-mail: <pquiroga@edu.ucm.es>.
} 
Los viajes de Paulo Freire a España (1984-1994) y la consolidación del ámbito académico de la...

para este estudo da presença do pensamento do pedagogo brasileiro nas Escolas de Educação da Espanha. A hipótese central que desenvolvemos neste trabalho é que, tomando como referência o trabalho de Freire, a pedagogia social conseguiu cavar seu próprio espaço no campo acadêmico da pedagogia na Espanha.

Palavras-chave: Paulo Freire. Espanha. Pedagogia Social. Educação Popular.

Abstract: Paulo Freire's ideas came to Spain since the 1970s. The first reception spaces were the popular education projects that were being promoted by progressive sectors of the Church and also by the Pedagogical Renewal Movements. Later Freire's ideas were received in the academic field of pedagogy in the late 1980s and early 1990s. Freire's trips to Spain between 1984 and 1994, four trips that are studied in detail in this article, are a key historical source for studying of the presence of the Brazilian pedagogue's thought in the Spanish Faculties of Education. The central hypothesis that we develop in this work is that taking Freire's work as a reference, social pedagogy managed to dig its own space in the academic field of pedagogy in Spain.

Keywords: Paulo Freire. Spain. Social Pedagogy. Popular Education.

\section{Introducción}

La dimensión social de la pedagogía, como ámbito de conocimiento propio de la Pedagogía en España, posee una dimensión bifronte. Por un lado, genera un corpus teórico propio y, por el otro, queda comprometida con la práctica por definición. Estas dos dimensiones conforman su identidad y también su mayor debilidad. Al proponer elaborar un corpus teórico entra en conflicto con la sociología de la educación. Cuando se inserta en el campo de la praxis el conflicto corporativo inicia con los trabajadores sociales. Si un autor ha contribuido durante la segunda mitad del siglo XX a resolver esta ambivalencia epistemológica ha sido Paulo Freire. Su obra es un referente en la defensa del espacio que corresponde a la Pedagogía Social en el contexto académico.

Un número importante de investigaciones han abordado en perspectiva histórica el proceso de consolidación en el ámbito de la pedagogía social en España (ÙCAR, 2018; CARIDE; ORTEGA, 2015; MÍNGUEZ ÁLVAREZ, 2004; FERMOSO ESTÉBANEZ, 2003; PÉREZ SERRANO, 2002;). Estos estudios tienden a poner de relieve la influencia alemana, francesa, inglesa o norteamericana que resultó clave en distintos momentos históricos para ir cavando el espacio propio de la pedagogía social en el contexto universitario. Apenas hay referencias a la influencia latinoamericana, a pesar de que existen estudios que han reivindicado desde América Latina esta vía de análisis (SILVA, 2016), Una influencia que en este trabajo consideramos clave y cuyo análisis ha de tener muy presente el pensamiento de Paulo Freire, así como la presencia que el pedagogo brasileño tuvo en espacios académicos españoles entre 1984 y 1994, y que se concretó en cuatro viajes con destino a Salamanca, Madrid y Barcelona, donde viajó en dos ocasiones.

Este es precisamente el punto de partida de este trabajo que centra su atención en los cuatro viajes de Freire a España por invitación de tres instituciones de educación superior: la Universidad Pontificia de Salamanca (UPSA) en 1984, la Universidad de Barcelona (UB) en 1988 y 1994 y la Universidad Complutense de Madrid (UCM) en 1991. Las fuentes históricas de referencia para este artículo son los textos que Freire discutió y presentó en estos espacios académicos, las cuales han sido localizadas en libros publicados, revistas académicas y videos en internet. Todos los cuales son citados con precisión en el artículo. Asimismo se incluye una fotografía inédita de su paso por la Facultad de Educación de la UCM encontradas en el Archivo General de esta universidad, junto con otra fotografía de su viaje a Salamanca. A nivel metodológico la investigación se sitúa en el campo propio de la Historia intelectual de la educación, en la medida en que estudia la recepción y circulación de ideas pedagógicas. De ahí que las preguntas que se van a responder en este trabajo encuentran fundamento histórico y también recorrido teórico: ¿Qué se sabe hasta el momento de la recepción de las ideas de Paulo Freire en España? ¿Qué fue lo más destacado de los cuatro viajes

Práxis Educativa, Ponta Grossa, v. 16, e2115143, p. 1-18, 2021

Disponível em: < https://www.revistas2.uepg.br/index.php/praxiseducativa> 
que Freire realizó a España entre 1984 y 1999? ¿En qué medida la presencia de Freire en tres universidades españolas contribuyó a reivindicar el espacio académico de la pedagogía social?

La relevancia que la obra de Freire a nivel internacional, cuyo paso por España se analiza en este articulo, ha sido recientemente recocida en la prestigiosa Encycopedia of Educational Theory and Philosophy:

\begin{abstract}
En su influyente libro -Pedagogía del oprimido (1970)- desarrolló las implicaciones de su visión de la naturaleza humana, haciendo de la historicidad el punto de inicio no solo de la esencia de los seres humanos sino también de la pedagogía emancipadora. Su teoría y pedagogía han influenciado movimientos de justicia social en cada continente, y han impactado en las prácticas en el aula entre los educadores que buscaban un enfoque humanístico de la enseñanza y el aprendizaje. (GLASS, 2014, p. 336).
\end{abstract}

\title{
Freire en España: aproximaciones a la recepción de sus ideas desde la historia de la educación
}

La obra de Freire toma como referencia el marco político y social de América Latina. Sus primeras experiencias educativas en los años cincuenta y sesenta en Brasil, se completó con frecuentes viajes por la geografía latinoamericana. En este sentido Freire se presenta como un exponente de primer orden para la pedagogía de la región y un referente del compromiso político y social que muchos intelectuales llegaron a adquirir a lo largo de la segunda mitad del siglo XX.

Con todo, restringir el impacto que la obra de Freire tuvo al contexto latinoamericano es un error. Es conocido que sus ideas llegaron mucho más lejos. Se puede observar en este sentido la relación que los lectores norteamericanos y europeos "establecieron entre la alienación histórica de la sociedad latinoamericana y la que experimentaba el ser humano moderno en las regiones más industrializadas del mundo" (IGELMO ZALDÍVAR, 2013, p. 23). A partir de los años ochenta cabe destacar la recepción de su obra en Estados Unidos promovida por quienes estaban trabajando en la pedagogía crítica norteamericana. También sus ideas llegaron a Europa, en concreto en países como Italia o Francia sus tesis fueron estudiadas y puestas en práctica.

Centrando la cuestión en las ideas de Freire en España, es posible identificar dos espacios de referencia en los años cincuenta, sesenta y setenta del siglo XX que han sido estudiados por historiadores de la educación. Ambos convergen en el tiempo, aunque representan dos marcos de recepción en parte diferenciados. El primero queda constituido por sectores de la Iglesia católica que en su compromiso con una pastoral social y educativa, en los años posteriores al Concilio del Vaticano II, tomaron como referencia las ideas de Freire para impulsar proyectos ligados a la educación popular especialmente en contextos urbanos. El segundo espacio de recepción está ligado a grupos de maestros que ya en los años finales de la dictadura de Franco, y también en los años posteriores a la muerte del dictador en 1975, encontraron en la obra de Freire un referente para la transformación radical del sistema educativo. Ambos espacios de recepción se adelantaron con relación al mundo académico español de la pedagogía, que hasta finales de los años ochenta y principios de los noventa, en buena medida gracias a una nueva generación de académicos cuyos trabajos educativos incluían una perspectiva más social, no prestó apenas atención a la figura intelectual de Freire.

A finales de los años cincuenta y principios de los años sesenta los suburbios de Madrid se configuran como enclaves de gran importancia en lo que a la historia de los movimientos urbanos se refiere, incluso en un plano internacional (CASTELLS, 1977). En condiciones de penuria económica, represión política y marginalización social, se constituyeron asociaciones vecinales que desde la periferia madrileña contribuyeron a la deslegitimación de la dictadura. Estas asociaciones

Práxis Educativa, Ponta Grossa, v. 16, e2115143, p. 1-18, 2021

Disponível em: < https://www.revistas2.uepg.br/index.php/praxiseducativa> 
funcionaron como "escuelas de ciudadanía" cuyo principal logro fue "mantener una inusual autonomía organizativa así como una duradera constancia en la movilización" (PÉREZ QUINTANA; SÁNCHEZ LEÓN, 2008, p. 14). Muchos de estos espacios con el tiempo se constituyeron como escuelas populares, cuya expansión se produjo entre 1970 y 1980. En estos proyectos "el método de enseñanza de Freire para la enseñanza popular de la lectura y la escritura fue adaptada al contexto de los suburbios usando 'palabras generadoras' como 'apartamento', ‘desempleo' o ‘barrio”’ (DEL POZO ANDRÉS; RABAZAS ROMERO, 2006, p. 241).

Respecto al rol que desempeñaron sectores de la Iglesia católica en España en el proceso de recepción de las ideas de Freire, es preciso traer a colación el terreno intelectual sembrado por jesuitas como Juan Soler de Morell, José María de Llanos o Enrique de Castro. Los dos primeros sentaron las bases de una educación popular pionera en España que tomó como referencia los lineamientos centrales de la Nouvelle Théologie y el apostolado social de vanguardia promovido por el movimiento de los sacerdotes obreros (SIEFER, 1965). Soler de Morel puso en marcha en 1943 los institutos seculares denominados "Hermandad de Cristo Trabajador", una organización con clara vocación obrera que tomó distancia de la línea paternalista hegemónica de la Iglesia española (SEGURO, 2005).

Por su parte, José María de Llanos, en el inicio de los años cincuenta, puso en marcha una pedagogía social a partir de tres grandes proyectos desarrollados en la Universidad de Madrid: el Colegio Mayor Santa María del Campo, el Colegio Mayor Antonio Rivera y el ambicioso Servicio Universitario del Trabajo (SUT) (IGELMO ZALDÍVAR; JOVER OLMEDA, 2019). Especialmente significativo fue el SUT que contó con la participación entre 1952 y 1969 de 13.254 estudiantes universitario que se involucraron en proyectos de servicio social comunitario en diferentes regiones de toda España. Estas experiencias de fundamento educativo suponían relacionar directamente la pedagogía con el medio social, precisamente en un tiempo en el que la pedagogía experimental prevalecía en el campo académico. A partir de 1954 nuevos proyectos educativos de gran calado social fueron promovidos por el Padre Llanos en el barrio del Pozo del Tío Raimundo en el Sur de Madrid. Siguiendo la estela de Soler de Morell y el Padre Llanos, el jesuita Enrique de Castro pondría en marcha proyectos en la línea de una pastoral educativa y social. Con el Concilio Vaticano II finalizado los proyectos que impulsó Castro fueron Traperos de Emaus, Madres contra la Droga, Escuela de Educadores y la Coordinadora de Barrio. Como explícitamente reconoció sus trabajos se centraban en "la promoción cultural en todos los niveles y en todas las edades, siempre con ese sentido crítico en una línea adaptada de la pedagogía de Freire" (CASTRO, 1997, p. 40).

Respecto al segundo espacio de recepción, Tamar Groves, en un artículo publicado en la revista Paedagogica Historica en 2011, ha estudiado con detalle el modo en que los primeros grupos de maestros españoles comenzaron a integrar las ideas de Freire para su desempeño pedagógico y sus reivindicaciones sociales en los años setenta. Su punto de partida es que durante los años finales de la dictadura franquista y los primeros de la transición a la democracia a partir de 1975, surgieron grupos de maestros en toda España cuya aspiración era democratizar el país mediante la transformación radical del sistema educativo. Según lo expuesto por Groves: "Estos grupos fueron destacados consumidores de publicaciones críticas que inundaron España después de años de censura y Paulo Freire se convirtió en una de sus más importantes fuentes de inspiración" (GROVES, 2011, p. 702-703).

Lo que Groves ha planteado en su estudio de la recepción de Paulo Freire, es que es posible trazar el impacto que sus ideas tuvieron en la consolidación de la identidad militante de los maestros y su compromiso con la realidad social española del momento. Al tiempo, la implementación del método de alfabetización de Freire que se llevó a cabo principalmente en la red de proyectos de

Práxis Educativa, Ponta Grossa, v. 16, e2115143, p. 1-18, 2021

Disponível em: < https://www.revistas2.uepg.br/index.php/praxiseducativa> 
educación popular y de escuelas de adultos que operaban en Madrid y Barcelona, fue un aliciente para vincular proyectos pedagógicos concretos con las aspiraciones de transformación social que emanaban de las clases populares. Las ideas de Freire quedaron insertas en proyectos culturales que lideraron los movimientos organizados de maestros y que conformaron toda una subcultura de participación democrática (GROVES, 2011). En el caso concreto de Barcelona, a partir de 1971, proyectos como las Escuelas de Adultos, las Escuelas de Alfabetización, los Círculos de Cultura o la Escuela Social, fueron espacios done las ideas de Freire tuvieron un impacto más que relevante. En buena medida "el pensamiento marxista de Freire y su peculiar humanismo cristiano lo hicieron particularmente apto para convertirse en el referente teórico más importante de aquella pequeña revolución cultural” (BOTEY; FORMARIZ, 1989, p. 34-35).

Es necesario subrayar que las actividades emprendidas por los Movimientos de Renovación Pedagógica llegarían a tener un impacto destacado a nivel académico. Por un lado, promovieron la apertura de una serie de revistas que renovaron el discurso educativo académico e introdujeron nuevas ideas que no tardaron en tener eco en el debate universitario. Entre esta revistas destacaron Cuadernos de Pedagogia, Colaboración, Guix, Reforma de la Escuela, Infancia y Aprendizaje, Perspectiva Escolar y Acción Educativa (HERNÁNDEZ DÍAZ, 2011, p. 90). Del otro, fueron un caladero de futuros profesores de las Facultades de Educación que a partir de los años ochenta se constituyeron en la práctica totalidad de universidades españolas y que llevaron sus inquietudes pedagógicas y sociales al terreno del mundo académico.

\section{Los cuatro viajes de Freire a universidades en España}

\section{a) El viaje a Salamanca: noviembre de 1984}

En el otoño de 1984 Freire emprendió un viaje a Salamanca, en concreto a la UPSA. Era una invitación cursada por la cátedra San José de Calasanz, un espacio de debate académico gestionado desde 1981 por los padres escolapios dentro del centro académico, cuyo fin era invitar anualmente, cada mes de noviembre, a una personalidad relevante del campo de la educación. Para entonces se había generalizado el interés por los trabajos de Freire en importantes universidades del mundo. Baste mencionar que entre 1973 y 1979 había recibido el título de Doctor Honoris Causa por centros académicos como la Universidad Abierta de Londres (1973), la Universidad Católica de Lovaina (1975), La Universidad de Michigan (1978) y la Universidad de Ginebra (GADOTTI; TORRES, 2001, p. 35).

Como hemos señalado anteriormente, entre los espacios de recepción preferentes de las ideas de Freire en España estaban colectivos católicos que trabajaban en una línea pastoral social y educativa de vanguardia. No es casualidad, por tanto, que su viaje a una universidad española fuera a uno de los dos centros de titularidad privada que gestionaba la Iglesia Católica en España. Aunque la fecha oficial de la fundación como institución privada de la UPSA es 1940, cabe subrayar que su propósito fundacional fue restaurar en Salamanca los estudios de Teología y Derecho Canónico que fueron el motivo de la creación en 1218 del Studium Generale, germen de la posterior Universidad de Salamanca (GARCÍA Y GARCÍA; AUBACH GUIU, 1989).

En el momento de su viaje a Salamanca las ideas de Freire comenzaban a estudiarse como el punto de anclaje central de la pedagogía crítica que en los años ochenta y noventa tendría una gran repercusión en Estados Unidos. En 1981 Henry Giroux había publicado un importante artículo en la revista Cualitative Inquiry titulado "Paulo Freire's Approach to Radical Educaciontal Reform" en el que retomaba ideas de Freire (GIROUX, 1981a). Este artículo formaría parte de uno de los libros más influyentes en Estados Unidos en el ámbito de la pedagogía que llevó por título Ideology, Culture and the Process of Schooling publicado en 1981. En su trabajo defendía que la

Práxis Educativa, Ponta Grossa, v. 16, e2115143, p. 1-18, 2021

Disponível em: < https://www.revistas2.uepg.br/index.php/praxiseducativa> 
obra de Freire "contiene muchos conceptos e ideas teóricas que proporcionan los pilares para una pedagogía aplicable en la experiencia norteamericana” (GIROUX, 1981b, p. 128). Otros intelectuales de la educación en los Estados Unidos aportarían destacadas contribuciones en la línea de la pedagogía crítica abierta por Giroux, es el caso de Peter McLaren, Antonia Darder, Donaldo Macedo o Michael Apple.

Freire estuvo cuatro días en Salamanca, a donde llegó el 25 de noviembre de 1984. Fue Jorge Sans Vila, entonces decano de la Facultad de Pedagogía de la UPSA, quien estableció el contacto con Freire y consiguió finalmente convencerle para que realizara un largo viaje desde Brasil hasta España. Freire impartió dos conferencias y participó en un coloquio con estudiantes de la licenciatura de Pedagogía. El día 26 de noviembre dio la primera conferencia que giró en torno a la cuestión "¿Para qué educarnos?". El 27 de noviembre la segunda conferencia se centró en las "Virtudes de la educación". El día 28 de noviembre se organizó un coloquio sobre las dos ponencias impartidas en la que Freire respondió a preguntas de los asistentes. El mismo día 28 Freire se reunió con los estudiantes de la licenciatura de pedagogía. La fuente histórica de referencia para conocer el trabajo de Freire durante esos días es el libro Freire en Salamanca. Tres conferencias y una charla abierta con los alumnos (GARCIA MADRID, 2016a).

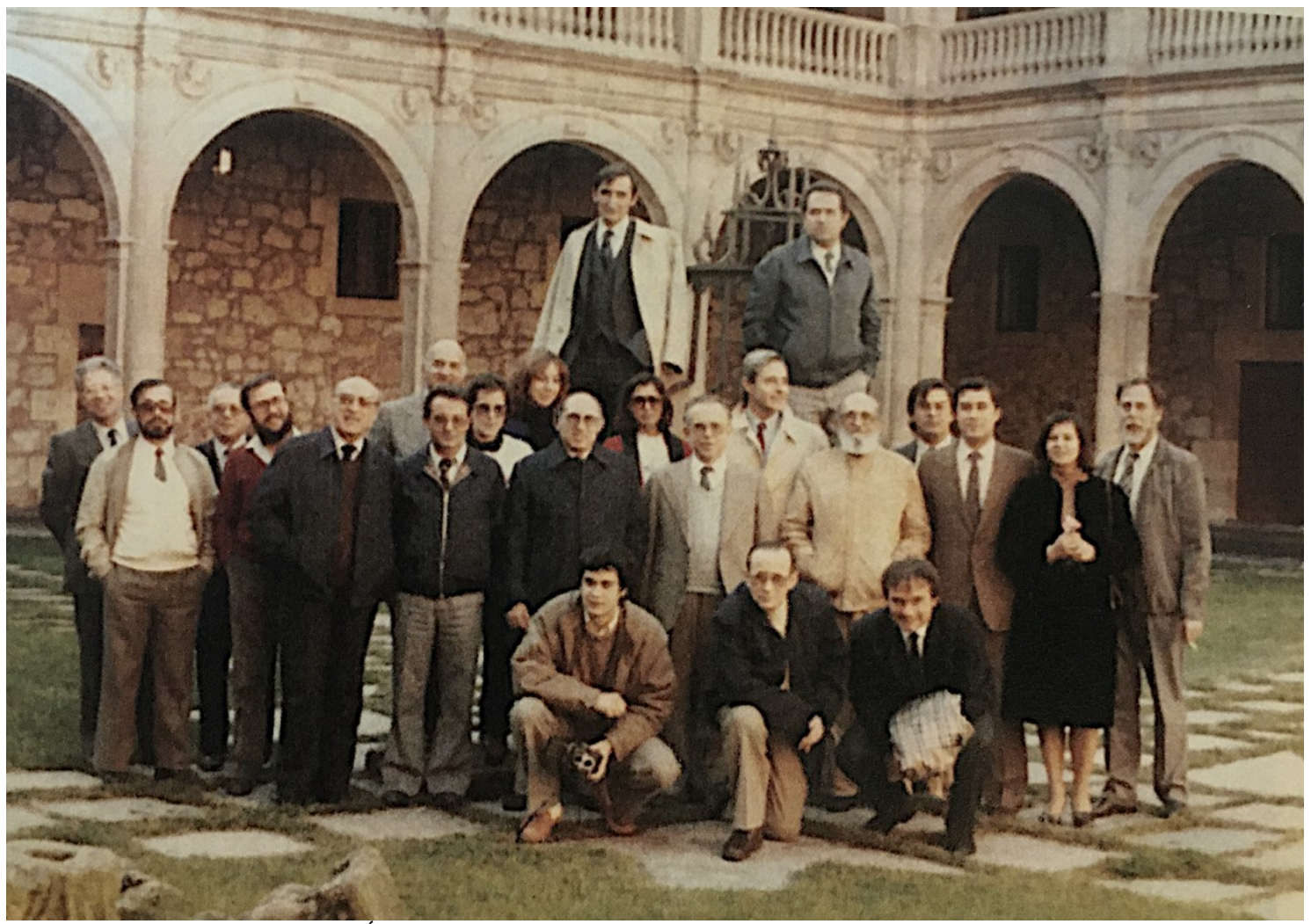

Fuente imagen: GARCÍA MADRID, 2016a, p. 120.

En la primera de las jornadas en Salamanca, con el espacio del seminario lleno de estudiantes, Freire fue presentado con unas palabras de agradecimiento del decano Sans Vila. En sus palabras quedó centrado el tema que el pedagogo brasileño desarrollaría en las tres jornadas de trabajo académico:

El local en que nos encontramos, siendo como es el mayor de la casa, ha resultado totalmente insuficiente para atender a la demanda de entradas, pese a no haberse hecho la más mínima propaganda. Y las negativas dadas estas últimas semanas para que el

Práxis Educativa, Ponta Grossa, v. 16, e2115143, p. 1-18, 2021 Disponível em: < https://www.revistas2.uepg.br/index.php/praxiseducativa> 
profesor Freire se trasladara a otras ciudades de España y a otras entidades de Salamanca han sido numerosísimas. Paulo Freire ha venido de avión en avión. Lo recogieron ayer en Barajas unos antiguos alumnos de esta Facultad de Pedagogía y le devolverán a Barajas el miércoles tan pronto como termine su conferencia (...) Los presentes todos, desean que usted les hable de "¿Para qué educarnos?". No como un libro. Los suyos están traducidos y se pueden leer. Sino como lo que usted es: Paulo Reglus Neves Freire, el hijo de Joaquim Temístocles Freire y Edeltrudes Neves Freire, el sobrino de Luzgardes Neves, el padres de Magdalena Cristina, Fátima, Joaquim y Luzgardes... y el abuelo de siete nietos. (SANS VILA, 2016, p. 20).

En las dos conferencias de los días 26 y 27, Freire adoptó un tono académico. Abordó de forma precisa cuestiones que ya habían sido desarrollados en sus más importantes libros años atrás, como eran la dimensión política de la educación, la vivencia de la práctica educativa o la relación entre educación y poder. A la pregunta que centró su primera ponencia, esto es "¿Para qué educarnos?", Freire terminó respondiendo en clave ontológica. Desde su perspectiva: "la educación es una respuesta necesaria a una dimensión fundamental humana, yo diría hasta de la ontología humana" (FREIRE, 2016a, p. 36). Con respecto a su ponencia en torno a las virtudes del educación del día 27, Freire fue enumerando y desarrollando en clave teórica las virtudes de los educadores. Destacó en su presentación como virtudes propias del educador comprometido con la transformación de la realidad: la humildad, la amorosidad, la virtud de vivir, la tensión entre la conciencia y el mundo, la asunción de la ingenuidad del educando, la coherencia, la tolerancia, la virtud de ir aprendiendo con la práctica y finalmente la virtud de seguir las reglas culturales del lugar al que uno llega (FREIRE, 2016b).

El día 28 fue el momento en que Freire confrontó sus ideas con los asistentes. El diálogo no fue tan de provecho como las ponencias en la medida que era un "auditorio de convencidos" (GARCÍA MADRID, 2016b, p. 102). Lo sucedido en la tercera jornada ha sido descrito también por García Madrid que asistió a ambas actividades:

\begin{abstract}
Las ocasiones de confrontación directa que Freire brindó en el encuentro dialogado de la tercera conferencia y el encuentro con los alumnos no dieron, a mi entender, el fruto que merecía. Freire fue, al ofrecer estos encuentros abiertos, muy coherente consigo mismo y con el discurso que proponía. (...) Me parece que a profesores y universitarios la liebre se le escapó viva de Salamanca. Quizá porque Freire era entonces mucho Freire y lo que significaba pesaba aún más. (Ibíd., p. 103).
\end{abstract}

\title{
b) El viaje a Barcelona: febrero de 1988
}

El viaje a Barcelona que Freire realizó en febrero de 1988 supuso un evento académico de gran relevancia para la División de Ciencias de la Educación de la UB. Esta División era el aparato institucional que coordinaba principalmente tres espacios de referencia para la formación de profesores y educadores en Cataluña, es el caso de la Facultad de Pedagogía, la Escuela de Formación de Profesorado de EGB y el Instituto de Ciencias de la Educación. Era la primera vez que una universidad española otorgaba el Doctorado Honoris Causa al pedagogo brasileño. Una generación de profesores que iniciaban entonces sus carreras académicas supieron ver la oportunidad y pusieron todo su esfuerzo para que la presencia de Freire contribuyera al afianzamiento de la Facultad de Pedagogía y los centros de formación del profesorado en el contexto universitario. Como señaló Ramón Flecha, que por entonces era profesor de Sociología y Director del Posgrado en Educación de Adultos, durante los meses previos tanto profesores como estudiantes y personal de administración y servicios pusieron todo su empeño y energía en organizar el homenaje. El propio Instituto de Ciencias de la Educación organizó un seminario en el que se estudió el conjunto de su obra. También desde la universidad se promovió "la campaña popular 'Freire en Catalunya' junto con los movimientos socioeducativos que están aplicando aquí (en Barcelona) su metodología” (FLECHA, 1989a, p. 11).

Práxis Educativa, Ponta Grossa, v. 16, e2115143, p. 1-18, 2021

Disponível em: < https://www.revistas2.uepg.br/index.php/praxiseducativa> 
El día antes de ser investido como Doctor Honoris Causa, el 1 de febrero, Freire se trasladó a la Escuela de Formación de Profesorado de E.G.B. (Educación General Básica) dependiente de la UB. Allí impartió la conferencia titulada "La Práctica Educativa". Sin duda este fue el acto académico más importante en el que participó en su segundo viaje a España. En el contexto del cambio generacional que estaban liderando entonces jóvenes profesores del ámbito de la pedagogía en Cataluña, es el caso de Jaume Trilla, Ramón Flecha o Jorge Larrosa, quedaron expuestas alguna de las ideas de base que definía a esta generación de pedagogos. Freire señaló que "es imposible que se enseñe, por ejemplo, una teoría pedagógica si no sé siquiera acercarme al objeto sobre el que hago teoría" (FREIRE, 1989a, p. 94). Lo cual, precisó, exige "una cierta comprensión del acto mismo de conocer, esto es, cuál es el rol de la subjetividad que intenta aprehender y conocer, que es conocer" (Ibíd.). Recalcó Freire que "más allá de su rol de político y de agente de conocimiento, más allá de su rol de artista, el educador, los educadores participan de una práctica educativa que ha de ser respetada, que ha de ser cultivada" (Ibíd., p. 96).

El acto de investidura de Freire como Doctor Honoris Causa tuvo lugar por la tarde del 2 de febrero en el paraninfo de la Universidad de Barcelona en el icónico edificio histórico de la Universidad de Barcelona de estilo neorromántico. La presentación la realizó Jaume Trilla i Bernet, profesor del Departamento de Teoría e Historia de la Educación de la Universidad de Barcelona. En su discurso de presentación, Trilla i Bernet puso énfasis en el hecho de que una de las contribuciones más notorias de Freire estaba relacionada con que gracias a sus trabajos, la pedagogía social y en concreto la educación de adultos, han ganado su espacio propio en el mundo académico, aún cuando "tradicionalmente habían estado marginados tanto del ámbito de la pedagogía teórica y académica como del propio sistema educativo" (TRILLA I BERNET, 1989, p. 27).

No obstante, en su intervención en el paraninfo, Freire no ahondó en cuestiones de carácter académico. Comenzó señalando que en celebraciones de este tipo, por lo general, evitaba "hacer discursos formales o discursos no lo suficientemente formales sobre temas de mi campo de reflexión" (FREIRE, 1989b, p. 102). De tal forma, cuando tomó la palabra su alocución fue en realidad un acto de agradecimiento en primer lugar a Elza María Oliveira, su esposa que había fallecido en 1986: "Mis palabras son de agradecimiento ya que hablo de mi gratitud para con Elza, a quien debí y debo tanto y tanto, y que amé tanto y que nunca olvidaré. Ciertamente, con Elza aprendí un mundo de cosas, pero, sobre todo, aprendí que, cuanto más amamos, más podemos amar". (Ibíd., p. 102). En segundo lugar sus palabras de agradecimiento fueron para quienes le había acompañado en su proceso de educación permanente y no tanto en la práctica académica: "los trabajadores del campo y la ciudad de diferentes partes del mundo, los campesinos y trabajadores urbanos, hombres y mujeres con los cuales aprendí, y aprendiendo con ellos y ellas, e igualmente fui enseñado" (Ibíd., p. 102). En el diario catalán El Periódico se publicó el 3 de febrero una crónica de la ceremonia que iniciaba así:

Pasadas las siete y media de la tarde, el rector de la Universidad de Barcelona, Josep María Bricall, imponía a Paulo Freire el canónico birrete azul tras haberle hecho entrega del pergamino conteniendo el título de doctor "honoris causa", el anillo y los tradicionales guantes blancos en un paraninfo abarrotado de estudiantes que habían aplaudido largamente al autor de La educación como práctica de la libertad (EL PERIÓDICO, 1988).

$\mathrm{Al}$ año siguiente se publicó el primer número de la revista Temps d'Educació. La nueva revista era una publicación semestral que partía de la iniciativa de la División de Ciencias de la Educación de la UB. Este primer número fue un tributo a la figura de Paulo Freire cuya presentación estuvo a cargo de Ramón Flecha. En el monográfico escribieron Jaume Trilla i Bernet, profesor del Departamento de Teoría e Historia de la Educación de la UB, Jaume Botey, teólogo, académico y profesor de historia en la UB; Anfonz Formariz, presidente de la Asociación de Educación

Práxis Educativa, Ponta Grossa, v. 16, e2115143, p. 1-18, 2021

Disponível em: < https://www.revistas2.uepg.br/index.php/praxiseducativa> 
Permanente de Adultos; Claudio Lozano Seijas; profesor de Historia de la Educación en la UB y Peter Javis, profesor del Departamentos de Estudios Educativos de la Universidad de Surrey (Reino Unido). El monográfico incluía el texto que Freire presentó el 1 de febrero de 1989 en la Escuela de Formación de Profesorado de E.G.B. y el que leyó en la ceremonia de investidura en el paraninfo de la universidad. Este número especial cerraba con una entrevista que Ramón Flecha realizó a Freire durante su estancia en Barcelona.

\title{
c) El viaje a Madrid: diciembre de 1991
}

Freire recibió en 1991 su segundo homenaje en España como Doctor Honoris Causa en la UCM. Fue clave en este sentido el papel que jugó Antonio Monclús Estella, profesor del Departamento de Didáctica y Organización Escolar. Gracias a su mediación y sus buenos contactos con el entorno intelectual de Freire, fue posible defender su candidatura al rectorado de la UCM y conseguir que el entonces profesor de la Universidad de São Paulo fuera nombrado el primer Doctor Honoris Causa propuesto por la Facultad de Educación de dicha universidad. Monclús Estella había escrito en 1998 uno de los ensayos más brillantes publicados en España sobre el pensamiento de Freire titulado Pedagogía de la contradicción: Paulo Freire. En este trabajo se hacía alusión a las dificultades que habían enfrentado las las ideas de Freire para abrirse paso en el contexto académico español de la pedagogía y en especial en la UCM:

\begin{abstract}
Es significativo asimismo lo que sucedió con Freire y su obra en nuestro contexto español. Precisamente en el momento en que la popularidad de Freire se extiende a nivel internacional, en España su obra está prohibida, y lo está hasta prácticamente la llegada de la transición democrática. No solamente sus libros estaban censurados, sino que es muchos centros educativos, en alguna sección de Ciencias de la Educación de Universidades Españolas, estaba proscrito su nombre como posibilidad de investigación, tesinas, tesis doctorales, etc. De esta manera Freire era también en España uno de los símbolos de la lucha por las libertades y por el establecimiento de un régimen democrático, particularmente en el ámbito de la enseñanza. Paradójicamente, como sucedía en otros tantos casos, la censura sobre su obra, ejercida fielmente en tantos sectores, repercutía en otros como mecanismo de motivación para estudiarlo especialmente (MOCLÚS ESTELLA, 1988, p. 17).
\end{abstract}

En el marco académico de los estudios de pedagogía uno de los escasos textos que durante el franquismo habían explorado las ideas de Freire, fue el publicado en 1974 por Víctor García Hoz en la Revista Española de Pedagogía con el título "La educación liberadora según Paolo Freire". Víctor García Hoz era catedrático de Pedagogía Experimental y Diferencial de la UCM y una de las figuras principales para la pedagogía en su despliegue como ciencia en el campo académico durante el franquismo (BERNAL-GUERRERO, 1999; CANALES, 2019). Era este un texto crítico y escrito en un tono despectivo sobre la obra de Freire. Llegaba a calificar algunas de sus ideas de simplistas, disparatadas, contradictorias y elaboradas a partir recursos retóricos. Sobre las aportaciones de Freire a la educación desde la perspectiva de la teoría de la acción dialógica presentada en Pedagogía del Oprimido (FREIRE, 2005, p. 218-219), García Hoz concluía que "el diálogo y la relación del aprendizaje con la vida, son tan viejos como Sócrates y Séneca por citar nombres bien conocidos y doctrinas bien definidas" (GARCÍA HOZ, 1974, p. 163).

Conseguir que Freire fuera investido Doctor Honoris Causa por la UCM era un claro síntoma del cambio generacional y de enfoque pedagógico que se estaba produciendo en la que históricamente había sido la institución académica de referencia para la pedagogía académica en España. Baste mencionar que fue en 1904 cuando Manuel Bartolomé Cossío ocupó la Cátedra de Pedagogía Superior en la Facultad de Filosofía y Letras de la Universidad Central, actual UCM (RUIZ BERRIO, 2005). Asimismo fue en Madrid donde iniciaron su andadura la Escuela Superior de Estudios de Magisterio 1909 y la Sección de Pedagogía en 1932, ambos centros de referencia para la configuración de la pedagogía como un espacio académico propio (JOVER, 2019). Durante

Práxis Educativa, Ponta Grossa, v. 16, e2115143, p. 1-18, 2021

Disponível em: < https://www.revistas2.uepg.br/index.php/praxiseducativa> 
la dictadura fue el Instituto San José de Calasanz de Pedagogía el que a partir de 1941, y con el ya mencionado Víctor García Hoz como director, relanzó desde una perspectiva experimental la investigación en pedagogía.

La Facultad de Educación de la UCM era, en buena medida, el espacio que marcaba una continuidad histórica directa con todas estas iniciativas académicas del ámbito propio de la pedagogía en España. De hecho, su constitución definitiva Facultad de Educación se había producido apenas un par de meses antes del viaje de Freire como resultado del Real Decreto 1456/91 del 27 de septiembre de 1991. Este Real Decreto propició la fusión dentro de una misma facultad de la Sección de Ciencias de la Educación de la Facultad de Filosofía y Ciencias de la Educación, la Escuela Universitaria de Profesorado de Educación General Básica María Díaz Jiménez, la Escuela Universitaria Pablo Montesino y el Instituto de Ciencias de la Educación. Todas estas instituciones educativas juntas, por su dimensión, conformaron a partir de 1991 la Facultad de Educación con más estudiantes y profesores de España (IGELMO ZALDÍVAR, 2019, p. 53$54)$.

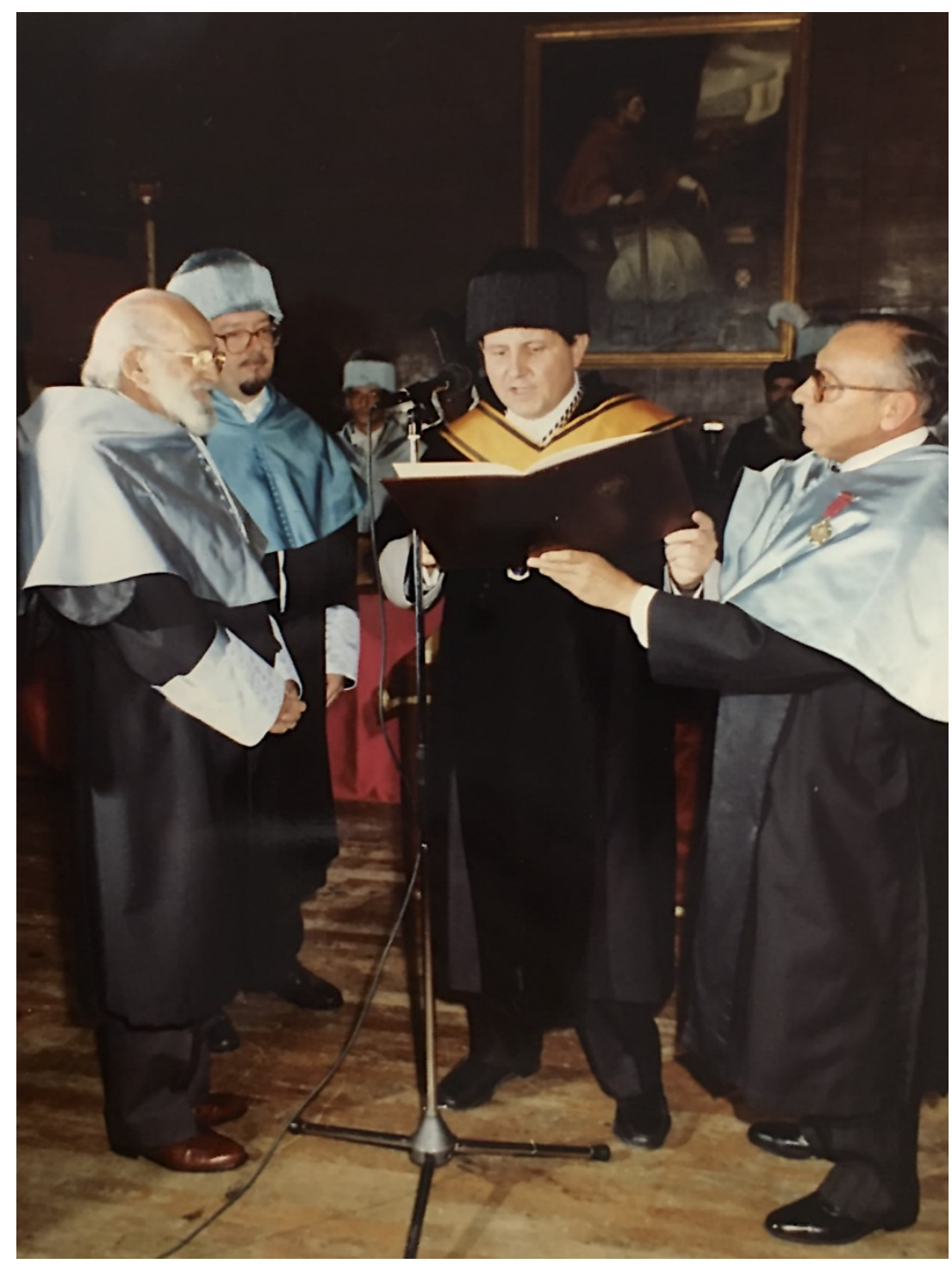

Fuente imagen: Archivo General de la UCM, 1991.

Con seguridad Freire conocía la relevancia que la recién creada Facultad de Educación de la UCM tenía para la configuración del campo académico específico de la pedagogía en España. 
Así, en su discurso de investidura celebrado en el paraninfo del edificio histórico de la UCM, en la popular calle San Bernardo de Madrid, inició señalando que al recibir este homenaje tenía muy presente la medida en que han servido de apoyo "mis acciones o reflexiones en el campo de la pedagogía". Aunque fue un discurso breve, que inició abordando de forma deslavazada cuestiones como el valor de la esperanza en la educación o la historicidad de los seres humanos en el mundo, dejó un mensaje claro sobre la necesidad de tomar distancia de discursos pedagógicos que restringen la experiencia educativa a una ciencia de programación de medios y formas para hacer llegar el conocimiento a los sujetos. Ante esta pedagogía que había sido hegemónica en España hasta los años ochenta, y en frente de muchos de los académicos que la habían impulsado y que estaban en el paraninfo de la UCM escuchando, Freire señaló:

\begin{abstract}
Sin sueño y sin utopía, los únicos cambios posibles que la generación recién llegada al mundo imprimirían al legado de las anteriores serían relacionados con las "sobras" del mismo proceso de adaptación a lo que habría encontrado. La negación del sueño y de la utopía, la defensa de la muerte de la historia y de las ideologías que pregonan algunos discursos 'modernizadores', significan la reedición, con un disfraz diferente, de la comprensión mecanicista de la historia, que negando en ella el papel fundamental de la subjetividad, termina por negar la libertad humana. (...)

La modernidad, exagerada en la defensa de sus certezas, a la que la llamada rigurosidad científica dio una gran aportación, está siendo hoy, felizmente, alterada. La postura científica y rigurosa hoy, en la posmodernidad, es exactamente la que niega la absolutización del conocimiento y reconoce su historicidad. La ciencia no es un 'a priori' de la historia, los hallazgos de los científicos son históricos y tienen, por eso mismo, historicidad. El conocimiento no es. Está siendo. Estas consideraciones hablan de como estoy, cada vez más, pensando y actuando como educador, como político, en cuanto educador y también en cuanto persona (FREIRE, 2017).
\end{abstract}

\title{
d) El viaje a Barcelona: julio de 1994
}

A raíz de su anterior viaje a Barcelona en 1988, Paulo Freire mantuvo el contacto con el profesor de la UB Ramón Flecha. Durante la estancia del pedagogo brasileño, Flecha, como quedó reflejado por él mismo en el monográfico dedicado a Freire en la revista Temps d'Educació en 1898, tuvo la ocasión no sólo de entrevistarle sino también de comer una paella con él, pasear por el Barrio Gótico de la Ciudad Condal y tomar café en una terraza. Para Flecha, la figura intelectual de Freire representaba "un símbolo" al ser "el pedagogo más conocido y carismático que queda vivo" (FLECHA, 1989b, p. 105). Lo cierto es que este profesor de la UB no sólo había estudiado la obra de Freire, sino que había puesto en práctica sus ideas en un conocido proyecto pedagógico en el que estuvo comprometido en los años setenta y ochenta en Barcelona. Se trataba de la Escuela de personas adultas La Verneda-Sant Martí fundada en 1978 (SÁNCHEZ AROCA, 1999; RACIONERO; SERRADELL, 2005).

El principal hecho que prueba que la relación con Ramón Flecha continuó en el tiempo, es que seis años después, con motivo del Congreso Internacional "Nuevas Perspectivas Críticas en Educación" celebrado en Barcelona los días 6, 7, 8 y 9 de julio de 1994, Freire volvió a viajar a España. Fue invitado a presentar la conferencia de clausura del congreso y para tal fin preparó un texto que tituló "Educación y participación comunitaria". Este evento académico contó también con la participación de referentes internacionales de las ciencias sociales como Manuel Castells, catedrático de Planificación y de Sociología de la Universidad de Berkeley; Henry A. Giroux de la Universidad Penn State; Donaldo Macedo de la Universidad de Massachussets; Paul Willis, catedrático de la Universidad de Wolverhamptom y el propio Ramón Flecha de la UB. Los textos con las ponencias presentadas fueron recogidos en un libro publicado en el mismo año del congreso (CASTELLS, FLECHA, FREIRE, GIROUX, MACEDO WILLIS, 1994).

Práxis Educativa, Ponta Grossa, v. 16, e2115143, p. 1-18, 2021

Disponível em: <https://www.revistas2.uepg.br/index.php/praxiseducativa> 
El texto que escribió Freire con el título "Educación y participación comunitaria" ponía énfasis en la idea de que la educación es una dimensión necesaria de la práctica social. La práctica social, desde su perspectiva, era la tensa relación entre lo heredado o lo recibido del contexto que es creado y recreado por cada ser humano. A continuación profundizó en nociones básicas de la educación progresista y despliegue en contextos democráticos por medio de la participación. A este respecto subrayo que la participación, en cuanto a ejercicio de la voz propia en el terreno social de la ciudadanía, se encuentra en relación directa con la práctica educativo-progresista. Freire señaló que "para los autoritarios, la democracia se deteriora cuando las clases populares están demasiado presentes en las escuelas" (FREIRE, 1994, p. 95). Siguiendo su texto, una estrategia posible para el fomento de la participación populares era el desarrollo de estructuras ligeras y descentralizadas que permitían que alumnos, profesores, madres y padres y toda la comunidad escolar se involucrasen en la experiencia democrática popular que en última instancia garantizaba la rapidez y eficacia de la acción gubernamental.

El último día del congreso, el 8 de julio de 1994, Paulo Freire fue el encargado de clausurar el congreso. La conferencia fue grabada y supone un documento histórico de gran valor. Para su presentación, Freire decidió separarse del texto de su ponencia. Según señaló en el inicio de su intervención consideraba que "cerrar una reunión debe significar mucho más abrir una temática" (FREIRE, 2012, min. 5:50-5:52). Y eso fue lo que hizo a partir de un discurso brillante en el que contextualizó muchas de sus ideas pedagógicas en el marco del neoliberalismo. Comenzó haciendo referencia a la experiencia de muchos educadores en los años sesenta que partieron de cierta "ingenuidad pedagógica" (Ibíd., min. 6:51). En esos años, los educadores integraron y desarrollaron "la esperanza en la fuerza instrumental de la educación como si ella pudiera ser, en un estilo idealista, la que lleva a las transformaciones sociales (Ibíd., min. 7:20-7:29). Ya en los años setenta "se inaugura una fase de pesimismo pedagógico" (Ibíd., min. 7:37-7:39). Y en los años ochenta aparece "una visión más crítica" (Ibíd., min. 8:50-8:53) en la medida que se llegó a una posición más dialéctica en la que se asume la reproducción, pero se percibía que dentro de la práctica de la escuela estaba la posibilidad de trabajar contra la reproducción ideológica en una perspectiva "desmitificadora de la reproducción ideológica" (Ibíd., min. 10.05-10:8). Finalmente para Freire en los noventa "la educación empieza a ser afirmada como indispensable, la llave para las transformaciones sociales", pero con el matiz de "que la historia es casi una no historia en base a discursos neoliberales” (Ibíd., min. 12:00-13:00).

Para Freire era fundamental señalar que el escenario global neoliberal estaba teniendo ya en los años noventa un impacto directo en la educación. Se decía, además, dentro de este nuevo marco discursivo que el tiempo de las ideas de Freire se había acabado, en la medida que "de lo que hablaba ya no tiene sentido" (Ibíd., min. 17:03-17:06). Y es que la idea central de sus trabajo era que "la educación debería ser un permanente esfuerzo crítico de desocultación de verdad, pero ahora ya no hay verdades, hay funciones diferentes" (Ibíd., min. 17:30-18:30). La educación bajo la lógica del neoliberalismo "no tendría que enseñar nada, simplemente el saber tecnológico y técnico del que se necesita para ser eficaz" (Ibíd., min. 20:00-20:55). No hay necesidad en este contexto de desvelar una realidad concreta, por eso denunció Friere que "en lugar de soñar con la humanización, de luchar para transformar una sociedad perversa, lo que importa es ver cómo aumenta la producción, pero no se discute a favor de quienes y contra quienes se aumenta la producción" (Ibíd., min. 21:05-21:38).

Friere finalizó su ponencia resaltando las principales ideas expuestas y recuperando también el pensamiento de un filósofo español como Miguel de Unamuno:

Yo creo que una de las tareas nuestras es disminuir la distancia entre las realidades duras y el sueño de mejorar el mundo. Lo que me parece imposible es que aceptemos ser hombres y mujeres, seres de la adaptación al mundo. Y es esta ideología que está por

Práxis Educativa, Ponta Grossa, v. 16, e2115143, p. 1-18, 2021

Disponível em: < https://www.revistas2.uepg.br/index.php/praxiseducativa> 
dentro de la filosofía e ideología política del discurso neoliberal, que nos debemos adaptar. Y es por esta razón que el discurso neoliberal es tan fatalista, profundamente fatalista, queriendo dar la impresión de que es actual. Es como si les dijera, mira, la fuerza de la nueva economía global, la fuerza de las organizaciones multinacionales que comandan la producción del mundo hoy, la fuerzas de una compañía que sabe cuál va a ser la producción de soja en Brasil en el próximo año por medio fotografía de satélite y controla el precio. El discurso neoliberal sugiere que con esto no hay nada que hacer sino aceptar. Yo acepto al científico que haga el análisis y muestre todas las dificultades, pero que en su análisis también diga que pelea contra esto. Es esto lo que yo quiero. $\mathrm{Y}$ es esto lo que yo les digo. Yo no vendría a España, sobre todo a España, a la España que me ha dolido, la España me duele dijo Unamuno. Yo no podría venir a un país que le dolía a un hombre como Unamuno para no decir que el mundo me duele. Y que yo no acepto de manera ninguna este fatalismo de que debemos usar una pedagogía que se porta bien, una práctica que piensa solamente en el quehacer de hoy, que no piensa el mañana, que no sugiere un conocimiento profundo de las cosas como se dan, por qué se dan, a favor de quienes se dan. Yo defiendo la misma pedagogía más radicalmente hoy que ayer, la pedagogía que ilumina, que desoculta y que no tiembla. Muchas gracias (Ibíd., min. 43:2346:40)

\section{Consideraciones finales}

Los cuatros viajes que emprendió Freire a España son en perspectiva histórica importantes acontecimientos para el giro social que emprendió el campo académico de la Pedagogía en los años ochenta y noventa en España. A pesar de que intelectuales como María de Maeztu había realizado ya al principio del siglo XX aproximaciones a la educación social desarrollada en Alemania por pedagogos como Natorp (GONZÁLEZ-GERALDO, 2019), y que dentro del principal órgano de divulgación académica de la pedagogía, la Revista de Pedagogía, en los años treinta se constituyó la Colección de Pedagogía Social y Política (MAINER BAQUÉ, 2010, p. 342), el auge de la Pedagogía Experimental a partir de los años cuarenta y cincuenta impidió el desarrollo académico de la Pedagogía Social en España. De forma muy marginal las las Secciones de pedagogía de Madrid (1944), Barcelona (1954) y Valencia (1966) incluyeron materias específicas de Pedagogía Social. En el caso de Barcelona y Valencia aparece la asignatura del "Pedagogía Social" en el cuarto curso. Mientras que en Madrid los contenidos de la pedagogía social quedan insertos en la "Pedagogía Racional (individual y social)" también en el cuarto curso (Ibíd., p. 823). Siendo en 1991 cuando se aprobaron las directrices generales de los planes de estudios del título de Diplomado de Educación Social (MACHADO, 2017, p. 659).

Los viajes de Freire pueden interpretarse, por tanto, en clave del definitivo despliegue que la Pedagogía Social inicia en España en los años ochenta. La base genealógica de este resurgir, como ya se ha mencionado en este trabajo, se encuentra en sectores progresistas de la Iglesia Católica que comienzan a implementar un apostolado social y educativo de vanguardia en las periferias de las principales zonas urbanas de España en los años cuarenta y cincuenta. No menos importante fue la relación entre educación, política y sociedad que articularon los Movimientos de Renovación Pedagógica. Ambos fueron espacios de recepción preferenciales de las ideas de Freire que antecedieron al impacto de sus ideas en el campo académico.

Por su parte, el impacto en el campo académico se produce a partir de los años ochenta y noventa. En estas dos décadas se publican notables trabajos que son en la actualidad una referencia para el análisis del modo en que los postulados de Freire circularon en el contexto español (FERNÁNDEZ FERNÁNDEZ, 2001; HERNÁNDEZ ARISTU, 1990; ESCOBAR AGUIRRE, 1989, BLANCO, 1982). También se publican monográficos sobre el pensamiento de Freire en revistas de referencia en España, como el publicado en 1998 en la Revista interuniversitaria de formación del profesorado. Aunque el estudio detallado de este fenómeno se escapa del foco de análisis de este

Práxis Educativa, Ponta Grossa, v. 16, e2115143, p. 1-18, 2021

Disponível em: <https://www.revistas2.uepg.br/index.php/praxiseducativa> 
trabajo, sí es posible concluir que Freire, con su presencia entre 1984 y 1994, contribuyó notablemente al fortalecimiento de la Pedagogía Social en España. Baste mencionar que el grado de Educación Social y los Master especializados en Educación Social que actualmente se imparten en la mayoría de Facultades de Educación en España, así como la especialización en Pedagogía Social del grado de Pedagogía, son en la actualidad elementos clave a los que se aferra la dimensión social de la pedagogía para justificar su espacio propio en el campo universitario.

Por su parte, la consolidación como ámbito delimitado dentro de la comunidad académica española se confirma gracias a órganos de expresión y divulgación tan relevantes como la publicación Pedagogía Social. Revista Interuniversitaria, que publicó su primer número en 1984 o Educació social. Revista d'intervenció socioeducativa que inició en 1995. La edición de revistas está relacionada con el impulso que experimentó este ámbito académico tras concretar su espacio autónomo con respecto a la Sociedad Española de Pedagogía. En este proceso de búsqueda de autonomía la fecha de 1985 es clave. Es entonces cuando inicia en España el encuentro anual "Seminario Interuniversitario de Pedagogía Social" que reunía a profesores universitarios que investigaban y publicaban dentro del ámbito específico de la pedagogía social en España.

En definitiva, los cuatro viajes a España posiblemente no fueron grandes hitos en la biografía personal de Freire. De hecho, apenas existen datos al respecto en los principales estudios biográficos del pedagogo brasileño. Por el contrario, para la consolidación del ámbito académico de la pedagogía y su dimensión social en España a partir de los años noventa, el paso de Freire por Salamanca, Madrid y Barcelona son fundamentales. Sorprende de hecho la escasa atención que estos viajes han suscitado entre los historiadores de la educación hasta el momento. Se considera, por tanto, que aquí reside el interés de este artículo.

\section{Referencias}

ARCHIVO GENERAL DE LA UCM. Investidura 'honoris causa' a Paulo Freire. Carpeta: ES-AGUCM 69/03-16, 1991.

BLANCO, R. La pedagogía de Paulo Freire. Ideología y método de la educación liberadora. Madrid: Zero, 1982.

BOTEY, J.; FORMARIZ, A. Presència de Freire en el naixement i l'evolució de les escoles d'adults a Catalunya. Temps d'Educació, n. 1, p. 33-59, 1989.

BERNAL-GUERRERO, A. Influencias en el pensamiento de Víctor García Hoz (1911-1998). Bordón. Revista de Pedagogía, v. 51, n. 2, p. 209-217, 1999.

CANALES, A. From Soul to matter: the new Spanish Francoist pedagogy's plunge into experimental pedagogy and the influence of Raymond Buyse. Paedagogica Historica, v. 55, n. 3, p. 451-469, 2019. DOI: https://doi.org/10.1080/00309230.2018.1560337

CASTELLS, M. Ciudad, democracia y socialismo. Las experiencias de las Asociaciones de Vecinos de Madrid. Madrid: Siglo XXI, 1977.

CASTELLS, M.; FLECHA, R.; FREIRE, P.; GIROUX, H.; MACEDO, D.; WILLIS, P. Nuevas perspectivas críticas en educación. Barcelona: Paidós, 1994. 
CARIDE, J. A.; ORTEGA, J. From Germany to Spain: origins and Transitions of Social Pedagogy through 20th Century Europe. In: KORNBECK, J.; ÚCAR, X. (ed.). Latin American Social Pedagogy: relaying concepts, values and methods between Europe and the Americans. Bremen: EHF Academic press Gmbb-Verlang, 2015. p. 13-27.

CASTRO, E. Dios es ateo. Madrid: Editorial Popular., 1997.

DEL POZO ANDRÉS, M. M.; RABAZAS ROMERO, T. Exploring new concepts of popular education: politics, religión and citizenship in the suburban schools of Madrid, 1940-1975. Paedagogica Historica, v. 42, n. 1-2, p. 221-242, 2006. DOI: https://doi.org/10.1080/00309230.2010.530282

EL PERIÓDICO. La Universidad de Barcelona rinde homenaje a Paulo Freire, el gran alfabetizado de adultos en Brasil. Publicado en diario El Periódico, 3 de febrero de 1998, p. 4, 1988.

ESCOBAR AGUIRRE, J. S. Paulo Freire: otra pedagogía política. Madrid: Universidad Complutense de Madrid, 1989.

FERMOSO ESTÉBANEZ, P. ¿Pedagogía social o Ciencias de la Educación Social? Pedagogía Social. Revista Interuniversitaria, n. 10 (segunda época), p. 61-84, dec. 2003.

FERNÁNDEZ FERNÁNDEZ, J. A. Paulo Freire y la educación liberadora. In: TRILLA, J. (coord.). El legado pedagógico del siglo XX para la escuela del siglo XXI. Barcelona: Grao, 2001. p. 313-342.

FLECHA, R. Presentació. Temps d’Educación, n. 1, p. 11-13, 1989a.

FLECHA, R. Conversant amb Paulo Freire. Temps d'Educación, n. 1, p. 105-109, 1989b.

FREIRE, P. La pràctica educativa. Temps d'Educación, n. 1, p. 91-100, 1989a.

FREIRE, P. Dicurs pronunciat a l'acte d'investidura com a Doctor Honoris Causa per la Universitat de Barcelona. Temps d'Educació, n. 1, p. 101-103, 1989b.

FREIRE, P. Educación y participación comunitaria. In: CASTELLS, M.; FLECHA, R.; FREIRE, P.; GIROUX, H.; MACEDO, D.; WILLIS, P. Nuevas perspectivas críticas en educación. Barcelona: Paidós, 1994. p. 83-96.

FREIRE, P. Pedagogía del Oprimido. 2. ed. México: Siglo XXI Editores, 2005.

FREIRE, P. Paulo Freire: perspectivas críticas en educación en Barcelona. ORRANTIA, J. S. Archivo de video: (56:25). Publicado en: 2012. Recuperado de https://www.youtube.com/watch?v=Uixem8Hah6U. Acesso en: 2 jun. 2012.

FREIRE, P. ¿Para qué educarnos? (Conferencia del día 26 de noviembre de 1984). In: GARCÍA MADRID, A. Freire en Salamanca. Tres conferencias y una charla abierta con los alumnos. Madrid: PCC, 2016a. p. 21-37. 
FREIRE, P. Virtudes del educador (Conferencia del día 27 de noviembre de 1984). In: GARCÍA MADRID, A. Freire en Salamanca. Tres conferencias y una charla abierta con los alumnos. Madrid: PCC, 2016b. p. 38-59.

FREIRE, P. Discurso Paulo Freire. Rizoma Freireano, 23. Disponible en: http://www.rizomafreireano.org/discurso-paulo-freire-es-siempre-23. 2017. Acesso en: 2 jun. 2020.

GADOTTI, W.; TORRES, C. A. Paulo Freire una biobibliografía. México: Siglo XXI Editores, 2001.

GARCÍA HOZ, V. La educación liberadora según Paolo Freire. Revista Española de Pedagogía, n. 126, p. 161-174, 1974.

GARCÍA MADRID, A. Freire en Salamanca. Tres conferencias y una charla abierta con los alumnos. Madrid: PPC, $2016 a$.

GARCÍA MADRID, A. Epílogo. Treinta años después. In: GARCÍA MADRID, A. Freire en Salamanca. Tres conferencias y una charla abierta con los alumnos. Madrid: PCC, 2016b. p. 101116.

GARCÍA Y GARCÍA, A.; AUBACH GUIU. M. T. La Universidad Pontificia de Salamanca: sus raíces, su pasado, su futuro. Salamanca: Universidad Pontificia de Salamanca, 1989.

GIROUX, H. A. Paulo Freire's Approach to Radical Educaional Reform. Curriclum Inquity, v. 9, n. 3, p. 127-141, 1981a. DOI: https://doi.org/10.2307/3202124

GIROUX, H. A. Ideology, Culture, and the Process of Schooling. Philadelphia: Temple University Press, 1981b.

GLASS, R. D. Freire, Paulo: pedagogy of the oppressed and critical pedagogy. In: PHILLIPS, D. C. (ed.) Encyclopedia of Educational Theory and Philosophy. United States: SAGE, 2014. p. 336-339.

GONZÁLEZ-GERALDO, J. L. Aportaciones de María de Maeztu a los inicios de la Pedagogía Social en España. Revista Complutense de Educación, v. 30, n. 1, p. 293-306, 2019. DOI: http://dx.doi.org/10.5209/RCED.57341

GROVES, T. Looking up to Paulo Freire: education and political culture during the Spanish transition to democracy. Paedagogica Historica, v. 47, n. 5, p. 701-717, 2011. DOI: https://doi.org/10.1080/00309230.2011.602348

HERNÁNDEZ ARISTU, J. Pedagogía del ser. Aspectos antropológicos y emancipatorios de la pedagogía de Paulo Freire. Zaragoza: Pensas de la Universidad de Zaragoza, 1984.

HERNÁDEZ DÍAZ, J. M. La renovación pedagógica en España al final de la transición. El encuentro de los movimientos de renovación pedagógica y el ministro Maravall (1983). Educació i Historia: Revista d'Història de l'Educació, n. 18, p. 81-105, jul./dec. 2011. DOI: https://doi.org/10.2436/20.3009.01.86 
IGELMO ZALDÍVAR, J. Paulo Freire y Jon Holt: de la educación liberadora a la libertad más allá de la educación. Educació i Historia: Revista d'Història de l'Educació, n. 21, p. 13-35, 2013.

IGELMO ZALDÍVAR, J. El Edificio de la Almudena de Ciudad Universitaria (1928-2019). In: IGELMO ZALDÍVAR, J.; FERNÁNDEZ ENGUITA, M. E1 Edificio de la Almudena de Ciudad Universitaria. La huella del pasado en tiempo de la hiperaula. Salamanca: FahrenHouse, 2019.

IGELMO ZALDÍVAR, J.; JOVER OLMEDA, G. Cuestionando la narrativa del aprendizaje servicio a partir de dos iniciativas de extensión social universitaria de orientación católica en la década de 1950 en España. Utopía y praxis latinoamericana, ano 24, n. 87, p. 151-162, oct./dec. 2019. DOI: http://doi.org/10.5281/zenodo.3464055

JOVER, G. Roots and Developments of Pädagogik in Spain. In: KUDLACOVÁ, B; RAJSKY, A. (ed.). Education and "Pädagogik". Philosophical and Historical Reflections (Central, Southern and South-Eastern Europe). Berlin: Peter Lang, 2019. p. 248-262.

MACHADO, E. R. A concepção de Pedagogia Social na formação dos Educadores Sociais na Espana. Práxis Educativa, Ponta Grossa, v. 12, n. 2, p. 657-681, 2017. DOI: http://doi.org/10.5212/PraxEduc.v.12i2.0021

MAINER BAQUÉ, J. La forja de un campo profesional: pedagogía y didáctica de la ciencias sociales en España (1900-1970). Madrid: Consejo Superior de Investigaciones Científicas, 2010.

MÍNGUEZ ÁLVAREZ, C. Evolución de la Pedagogía Social para consolidarse como disciplina científica. Pedagogía Social. Revista Interuniversitaria, n. 11, segunda época, p. 25-54, 2004.

MONCLÚS ESTELLA, A. Pedagogía de la contradicción: Paulo Freire. Nuevos planteamientos en educación de adultos. Barcelona: Anthropos, 1988.

PÉREZ QUITINA, V.; SÁNCHEZ LEÓN, P. Cuarenta años de movimientos ciudadanos. In: PÉREZ QUITINA, V.; SÁNCHEZ LEÓN, P. (ed.) Memoria ciudadana y movimiento vecinal. Madrid 1968-2008. Madrid: Catarata, 2008. p. 13-18.

PÉREZ SERrANO, G. Origen y evolución de la Pedagogía Social. Pedagogía Social. Revista Interuniversitaria, n. 9, segunda época, p. 193-231, dec. 2002.

RACIONERO, S.; SERRADELL; O. Antecedentes de las comunidades de aprendizaje. Educar, n. 35, p. 29-39, 2005.

RUIZ BERRIO, J. Manuel Bartolomé Cossío y los comienzos de los estudios de Pedagogía en la Universidad de Madrid. In: RUIZ BERRIO, J. (ed.) Pedagogía y Educación ante el siglo XXI. Madrid: Universidad Complutense de Madrid, 2005. p. 117-142.

SÁNCHEZ AROCA, M. Voices Inside Schools - La Verneda-Sant Martí: A School Where People Dare to Dream. Harvard Educational Review, v. 69, n. 3, p. 320-336, 1999. DOI: https://doi.org/10.17763/haer.69.3.gx588q10614q3831

SANS VILA, J. Presentación de Paulo Freire. In: GARCÍA MADRID, A. Freire en Salamanca. Tres conferencias y una charla abierta con los alumnos. Madrid: PCC, 2016. p. 18-20. 
SEGURO, J. F. La participación de los católicos en el movimiento obrero de Barcelona (1946-1978). Tesis (Doctorado en Història Contemporània) - Departament D'Història Contemporània, Universitat de Barcelona, Barcelona, 2005.

SIEFER, G. Los sacerdotes obreros (los hechos y las consecuencias). Barcelona: Nova Terra, 1965.

SILVA, R. Os fundamentos freirianos da Pedagogia Social em construção no Brasil. Pedagogía Social. Revista Interuniversitaria, v. 27, p. 179-198, 2016. DOI: https://doi.org/10.7179/PSRI 2016.27.09

TRILLA I BERNET, J. La relació teoría-pràctica en la pedagogía del Paulo Freire. Temps d'Educació, n. 1, p. 15-32, 1989.

ÚCAR, X. Pedagogía Social en Europa y América Latina: Diálogos e interacciones en el marco de lo común. In: DEL POZO SERRANO, F. J. Pedagogía Social en Iberoamérica: Fundamentos, ámbitos y retos para la acción socioeducativa. Barranquilla: Universidad del Norte-UNED, 2018. p. 3-33.

Recibido em: 20/04/2020

Versión corregida recibida: $12 / 10 / 2020$

Aceptado: $13 / 10 / 2020$

Publicado online: $15 / 10 / 2020$ 